\title{
AVALIAÇÃO DA PERCEPÇÃO DO ATO DE CUIDAR ENTRE CUIDADORES DE IDOSOS: ESTUDO QUALITATIVO
}

\footnotetext{
Jaqueline Rezende de Souza ${ }^{*}$; Marcos Vinícius de Oliveira ${ }^{2}$; Luiz Henrique Batista Monteiro²; Ivânia Vera ${ }^{3}$; Roselma Lucchese ${ }^{3}$

1. Bacharel em Enfermagem, Universidade Federal de Goiás (UFG), Regional Universitário de Catalão. Catalão-GO, Brasil. (jaquelinerezendesouza@gmail.com).

2. Bacharel em Enfermagem, Universidade Federal de Goiás (UFG), Regional Catalão - GO, Brasil.

3-Docente da Universidade Federal de Goiás (UFG), Regional Catalão. Catalão $\mathrm{GO}$, Brasil.
}

Recebido em: 08/04/2017 - Aprovado em: 10/06/2017 - Publicado em: 20/06/2017 DOI: 10.18677/EnciBio_2017A125

\begin{abstract}
RESUMO
As formas de enxergar e vivenciar a velhice são singulares, cada indivíduo reage de uma forma diferente, por suas características fisiológicas, emocionais e psicológicas, bem como espiritual. Estima-se que no ano de 2050, o público idoso chegará a dois bilhões, no mundo, sendo 100 mulheres idosas para cada 84 homens. Desta forma, a presença de cuidadores nas residências dos idosos vem sendo mais procurada e se tornado mais frequente. $O$ cuidador tem como função assessorar o idoso necessitado físico ou mentalmente, a desenvolver as práticas das atividades da vida diária e autocuidado. Sendo assim, este estudo objetivou avaliar a percepção do ato de cuidar entre os cuidadores de idosos do Sudeste Goiano. Os dados foram obtidos através de um questionário com a seguinte pergunta antes e após a atividade "Qual o significado do cuidado para você?". Os dados foram coletados em dezembro de 2015, tendo como requisito o público alvo ser $\geq 18$ anos e ser cuidador de idoso. Dentre as ações implementadas observou-se feedback positivo por parte dos participantes e anseio por outros momentos educativos e de continuação para esse público.
\end{abstract}

PALAVRAS-CHAVE: Cuidadores; Envelhecimento; Percepção.

\section{STUDY EVALUATIVE AND PERCEPTION OF THE ACT OF CARING BETWEEN THE SOUTHEAST GOIANO ELDERLY CAREGIVERS}

\begin{abstract}
The ways of seeing and experiencing old age are unique, each individual reacts in a different way, by their physiological, emotional and psychological as well as spiritual characteristics. It is estimated that by the year 2050, the elderly population will reach 2 billion in the world, 100 of which are elderly women for every 84 men. In this way,
\end{abstract}


the presence of caregivers in the homes of the elderly has been more sought after and more frequent. The caregiver's role is to advise the needy elderly physically or mentally, to develop the practices of activities of daily living and self-care. Thus, this study aimed to evaluate the perception of the caring act among caregivers of elderly people in Southeast Goiania. The data were obtained through a questionnaire with the following question before and after the activity "What is the meaning of care for you?". Data were collected in December 2015, with the target audience being $\geq 18$ years old and being a caregiver for the elderly. Among the actions implemented we obtained positive feedback from the participants and I look forward to other educational and continuation moments for this audience.

KEYWORDS: Aging; Caregivers; Perception.

\section{INTRODUÇÃO}

Calcula-se que para o ano de 2050, a população idosa será de dois bilhões de pessoas, no âmbito mundial, para cada 100 mulheres idosas há 84 homens. Quanto aos idosos longevos, ( $\geq 80$ anos ou octogenários), para cada 100 mulheres, há 61 homens em âmbito mundial (WHO, 2012). Nesse sentido, o envelhecimento é compreendido em dois aspectos a senescência, caracterizada pela diminuição da reserva funcional do indivíduo, e senilidade que diz respeito ao envelhecimento patológico, associado a doenças, traumas físicos ou psicológicos (CIOSAK et al., 2011).

Considerando o contexto da saúde dos idosos, identificam-se mudanças significativas no que tange a morbimortalidade, que tem diminuição na incidência de óbitos por doenças infectocontagiosas e o aumento da incidência de morte e doenças por condições crônicas (GAIOLI et al., 2012). Nesse sentido, a presença de cuidadores nas residências dos idosos tem se tornado mais frequente. O papel do cuidador é auxiliar o idoso impedido físico ou mentalmente, a desempenhar as tarefas práticas das atividades da vida diária e autocuidado (COELHO et al., 2013).

Têm-se dois tipos de cuidadores: cuidadores informais familiares (filhos, mães, esposas, entre outros) estes possuem algum tipo de vínculo com o idoso (ABREU et al., 2009). Já os cuidadores formais são os cuidadores de idosos, que são caracterizados como profissionais, os quais empregam suas habilidades competências para cuidar do outro (FARIAS \& SANTOS, 2012; GAIOLI et al., 2012). Nesse sentido, o presente trabalho objetivou avaliar a percepção do ato de cuidar entre cuidadores de idosos de um município da região central do Brasil.

\section{MATERIAL E MÉTODOS}

\section{Tipo e local do estudo}

Estudo descritivo exploratório de abordagem qualitativa, realizado durante uma ação de extensão interdisciplinar da Universidade Federal de Goiás Regional Catalão em dezembro de 2015. O curso foi constituído por docentes do curso de psicologia e a ação foi conduzida por docentes e discentes do curso de enfermagem e psicologia da referida universidade. A população alvo constituiu-se de 14 cuidadores de idosos em exercício da função formal/informalmente. Os critérios de inclusão, a saber: idade $\geq 18$ anos e ser cuidador de idoso. 


\section{Coleta de dados}

Para obtenção dos dados, os pesquisadores já devidamente treinados, dirigiram aos participantes, a seguinte pergunta antes e após a atividade "Qual o significado do cuidado para você?". Os dados foram coletados no mês de dezembro de 2015. Em razão de questões éticas, empregaram-se intitulação dos Entrevistados em distribuição número lógica (E1 a $E 14$ ) para divulgação das falas.

\section{Análise dos dados}

Para a análise das perguntas utilizou-se a análise temática de BARDIN (2009), sendo esta compreendida como um agrupamento de recursos metodológicos, que podem ser empregados em quaisquer discursos. A análise temática se constitui como sugere a autora, em buscar os "núcleos de sentidos", sendo estes incorporados no processo da comunicação, em que suas presenças constantes nas falas evidenciam algo para o objetivo analítico. A técnica de Bardin é desenvolvida em três fases, pré-análise, seleção das unidades de análise e processo de categorização respectivamente (BARDIN, 2009).

$\mathrm{Na}$ primeira fase realizou-se a pré-exploração do material, isto é, a leitura "flutuante". Segundo BARDIN (2009), a leitura "flutuante" visa propiciar o conhecimento do texto e das falas. Para tanto, foram executadas inúmeras de todo o material, buscando apreender, as idéias e os significados das falas.

$\mathrm{Na}$ segunda fase selecionaram-se as unidades de análise (ou unidades de significação). Na parecença dos objetivos a serem alcançados pela investigação. Logo, foi necessário realizar e construir os recortes das falas dos sujeitos, relatos e documentos. Tais recortes favorecem a identificação de unidades, que podem ser comparadas, e codificadas (BARDIN, 2009).

$\mathrm{Na}$ terceira e última fase houve a categorização, que denomina a classificação dos elementos do conjunto, o qual possibilita a diferenciação e, reagrupamento, segundo o gênero. Esse foi um processo que comportou duas etapas: a de inventário que isola os elementos e a de classificação que reparte os elementos. O primeiro objetivo da categorização fundamentou-se em fornecer, por meio da condensação das informações, a representação simplificada dos dados brutos (BARDIN, 2009).

Este estudo insere-se em uma pesquisa matriz aprovada pelo Comitê de Ética em Pesquisa da Universidade Federal de Goiás, protocolo número $523.834 / 14$ e respeitou a Resolução 466/2012. Todos os cuidadores de idosos que concordaram participar da pesquisa foram orientados quanto aos objetivos da pesquisa, bem como dos riscos e benefícios inerentes a participação, e assinaram o Termo de Consentimento Livre e Esclarecido previamente à coleta de dados e entrevista.

\section{RESULTADOS E DISCUSSÃO}

Dos 14 participantes do estudo, a maioria majoritária (92,9\%) era do sexo feminino, e com idade inferior a 50 anos, 78,6\% (11). Neste grupo, seis pessoas possuíam tempo de cuidador menor que cinco anos e oito possuíam tempo maior. Para fins de avaliação do impacto da intervenção na percepção dos cuidadores sobre o cuidado, foi questionado aos mesmos "Qual o significado do cuidado para você?" No início e após o término do curso. Da fala dos sujeitos, emergiram duas categorias de análise que serão discutidas a seguir. 


\section{A humanização do cuidado.}

No presente estudo notou-se que a primeira visão dos cuidadores entrevistados, está ligada a oferta de subsídios básicos para a manutenção da vida, como na fala a seguir:

[...] Eu me sinto muito feliz e esta preparando alimentos para eles e como se fosse para eu próprio para eu é uma satisfação. [...] E7

Para CIOSAK et al. (2011), o envelhecer é caracterizado por modificações sucessivas e inevitáveis, de modo natural, associados à idade e deste modo, o subsídio oferecido ao idoso tem como dever preservar a qualidade de vida, levando-se em consideração possíveis ações de promoção, resguardo, preservação e recuperação do seu estado de saúde.

Outros ainda atentam-se para o suporte emocional que a pessoa cuidada requer e relacionam sua atividade de trabalho aos sentimentos como amor e carinho como nas falas de E1, E4, E6, E8 e E10. Percebe-se que o cuidador é o sujeito designado para o cuidado com o idoso dependente, seja por apresentarse em condições limitadas psicológicas, físicas, entre outras, podendo ser remunerado ou não. É protagonista imprescindível no processo de recuperação e manutenção da saúde, além de auxiliar nas atividades do dia a dia do longevo sob condições de fragilidade, principalmente no que tange as ações de sentidos relacionados ao bem estar físico e mental, proteção, consolação, independência e autogoverno (ARAÚJO et al., 2013).

[...] Resumo do cuidado vem do amor paciência, carinho para estar certo que a gente ganha para isso, mas se não tiver isso não tem como. [...] E6

[...] É especial, dar carinho, amor, atenção, tudo na hora certinha e muito valor. [...] E4

[...] Amor que se não tiver não tem como cuidar o amor pelo próximo. Fazer tudo de bom e de melhor para a pessoa. [...] E1

[...] É gratificante oferecer o carinho cuidar é ver ele bem, feliz, tranquilo, calmo. [...] E8

[...]Cuido deles como se fosse meus filhos. [...] E10

Percebe-se que as ações referentes à prática do cuidado, por exemplo, ao realizar as atividades básicas como, alimentação, posicionamento, transporte, entre outros, provoca em seus familiares e/ou cuidadores dificuldades em saber lidar com determinadas situações, proporcionando um meio limitado ao cuidar da pessoa dependente acarretando na maioria das vezes constrangimento ao prestar os devidos cuidados, por serem de alta complexidade, dificultando a realização das mesmas (LANDEIRO et al., 2015).

ARAÚJO et al., (2013) chamam a atenção para as alterações emocionais e de humor que podem ocorrer entre os familiares, provocadas pelos cuidados que devem ser prestados ao idoso, essas modificações implicam em impactos de grande relevância no cotidiano dos cuidadores, como mudanças indispensáveis 
envolvendo apego, carinho, atenção, questões financeiras e de recursos, entre outros. Passa-se então por uma readaptação familiar, em que na situação um membro da família não está mais apto para realizar suas atividades particulares, a favor do outro. A fala de E11 demonstra que no grupo entrevistado, esse olhar voltado também a saúde do cuidador está presente.

[...] Manter equilíbrio moral e físico e psicológico do idoso e do cuidador pensar na lógica de facilitar as atividades com 0 idoso e manter com facilidade 0 relacionamento familiar. [...] E11

Diante do exposto, faz-se necessário projetar um vínculo entre a pessoa cuidada e o cuidador, promovendo uma assistência humanizada com um olhar integral para com o outro, sendo esperado um bom retorno em consequência do bom relacionamento entre ambos. Desta forma o cuidado prestado ao sujeito necessitado deve ser realizado abrangendo todo o contexto de vida dos mesmos, sendo indispensável que o profissional compreenda que o serviço prestado deve abarcar totalmente sua integridade de ser humano, não somente a responsabilidade em cuidar do processo patológico em questão (PEREIRA et al., 2014).

Ressalta-se que o cuidado humanizado ao idoso é determinado por uma relação entre cuidador/pessoa ocorrendo um feedback ao cuidado prestado de um indivíduo para o outro em circunstancia de dificuldade, que está voltada propositadamente, para proporcionar segurança, tranquilidade, conforto e bem estar físico e mental. Para que haja uma boa comunicação entre cuidador/idoso é preciso que ambos sejam acessíveis, atendendo as necessidades de escuta, envolvimento, desempenho e dando relevância a suas vontades, com a intenção de juntos elaborarem um plano de intervenções de cuidados. Deste modo o cuidador deve prestar seus cuidados de uma forma singular e humanística associado às técnicas e seu ciclo vital (SILVA et al., 2014). O participante E9 ilustra bem esse cuidado humanizado em sua fala.

[...] Depende da fase que o idoso está vivendo. O cuidado é uma forma de conduzir as atividades de vida e auxiliar nas atividades diárias. Oferecer amor devido a solidão. [...] E9

É importante lembrar, que o sujeito no decorrer da sua vida traz consigo uma bagagem de experiências as quais eles narram e reiteram suas lembranças no passar do tempo, sendo estes particularidades relevantes que não devem ser desconceituado ao tratar de dedicar- se ao outro, respeitando seus padrões, moral, preceitos, dando relevância a história falada e refalada, aos acontecimentos vividos no âmbito pessoal, expressando assim o olhar holístico que é almejado no atual contexto de atenção a saúde, investigando medidas juntamente com o composto familiar (SILVA et al., 2014).

\section{O despertar para a ciência do cuidar.}

GAIOLI et al., (2012) demonstram em estudo que associado ao crescente envelhecimento patológico, percebe-se que a procura por cuidado e cuidadores cresce de forma exponencial, necessitando assim de cuidadores com preparação e bagagem de conhecimentos específicos para oferecer cuidados. Os mesmos 
autores reforçam ainda que a apresentação de um cuidador capacitado para exercer sua função não é algo fácil de encontrar, sendo um cenário desafiador para o setor saúde, uma vez que, com o avanço da idade, o idoso torna-se mais dependente ao ter que realizar suas práticas do dia a dia. O progresso da doença pode ser inevitável ou não, sendo o cuidador responsável por ampliar seus conhecimentos, aperfeiçoando suas habilidades de cuidar. É importante afirmar que neste estudo, $10(71,4 \%)$ dos cuidadores entrevistados, não tiveram formação preparatória para esta função, corroborando com as conclusões de GAIOLI et al (2012).

Nesse contexto, os participantes da atividade de extensão (E1, E3 e E4), revelaram em suas falas o impacto positivo desse momento para eles:

[...] Novos conhecimentos sempre são muito importantes. Porque a pessoa que está cuidando do outro sempre precisar buscar novas informações, experiências, novas práticas. [...] E1

[...] Aumento de curiosidade para aprender mais, ter novas experiências profundas esse e meu objetivo capacitar mais e mais, compartilhar mais amor aos idosos, ouvir mais. [...] E3

\section{[...] Antes eu sabia menos só que agora aprendi mais estou tão feliz. [...] E4}

Verifica-se que existem alguns impasses e obstáculos confrontados na função do cuidador de idosos que possuem um nível de dependência elevado, porém, há a probabilidade de colher frutos satisfatórios para o profissional cuidador, o qual pode ser relacionado a um elevado ou diminuído poder de resiliência, sendo imprescindível o oferecimento de cursos preparatórios para a operacionalização de medidas de cuidados orientando sempre a adequação destes no que diz respeito ao ato de cuidar, relacionado a dependência gradativa dos longevos (GAIOLI et al., 2012).

[...] Este curso foi ótimo e eu aprendi coisas que não sabia e sempre que tiver essa oportunidade eu quero participar é um dia gratificante. [...] E8

[...] Continuo no meu objetivo, amor, carinho, responsabilidade, atenção, sendo assim saindo bem mais "extruída" aos meus cuidados sempre. [...] E6

[...] Preciso de repensar algumas formas de equilibrar melhor as condições psicológicas do idoso buscar pesquisar forma diferenciadas. [...] E11

Por fim, é visível que o ato de cuidar acarreta bons resultados e contentamento particular para cada profissional, aperfeiçoando o bom senso ao realizar as tarefas, um maior envolvimento com o outro, amor pelo que faz, exaltação, dignidade e capacidade para responder aos novos enfrentamentos, aperfeiçoando o vínculo com cada ser e com uma sensação de dever cumprido (GAIOLI et al., 2012).

[...] Hoje seria mais fácil, deu para pegar bem. Acredito que seria bem mais fácil. [...] E14 
[...] Eu aprendi que não sei nada da vida e preciso melhorar muito. [...] E10

[...] Eu achei importante o curso de capacitação de idoso agente aprendeu algumas dúvidas que temos ter paciência e admiração por cada dia de trabalho com idoso. [...] E7

\section{CONCLUSÃO}

O objetivo deste estudo foi avaliar a percepção de cuidadores de idosos sobre o ato de cuidar, nesta perspectiva, notou-se que os participantes possuíam previamente o olhar romantizado sobre o cuidado, descrito por falas que remetem ao amor e carinho no cuidado e que após a intervenção apresentaram uma fala mais consistente com um cuidado pautado em conhecimento e práticas seguras. É necessário considerar a precariedade de formação técnica dos participantes e ressaltar o reconhecimento da necessidade de um curso preparatório de cuidadores, por parte dos mesmos, de forma que estejam capacitados a desenvolver suas atividades com maior segurança sem prejuízo físico ou mental para si e para o cliente. Diante dessas conclusões sugere-se aos cuidadores que pratiquem educação permanente de acordo com suas disponibilidades, o que ocasionará em reflexos positivos na desenvoltura do papel de cuidador, de forma consciente e responsável abordando sempre questões fundamentais que englobam o cuidado, a humanização e qualidade de vida.

\section{REFERÊNCIAS}

ARAUJO, J. S.; VIDAL, G. M.; BRITO, F. N.; GONÇALVES, D. C. A.; LEITE, D. K.M.; DUTRA, C. D. T.; PIRES, C. A. A. Perfil dos cuidadores e as dificuldades enfrentadas no cuidado ao idoso, em Ananindeua, PA. Revista Brasileira de Geriatria e Gerontologia., v.16, n.2, p.149-158, 2013. Disponível em: http://www.scielo.br/pdf/rbgg/v16n1/a15v16n1.pdf. Acesso em: 12 set 2016.

ABREU, C. B.; RIBEIRO, M. I.; PIRES, N. R. Cuidando de quem já cuidou: o livro do cuidador. São Paulo: Atheneu; 2009.

BARDIN, L. Análise de conteúdo. 4 ed. Lisboa: Edições 70; 2009.

CIOSAK, S. I.; BRAZ, E.; COSTA, M. F. B. N. A.; NAKANO, N. G.R.; RODRIGUES, J.; ALENCAR, R. A.; ROCHA, A. C. A. L. Senescência e senilidade: novo paradigma na atenção básica de saúde. Revista da Escola de Enfermagem da USP, São Paulo, v. 45, n. spe 2, Dec. 2011.

COELHO, E. R.; SACERDOTE, D. dos S.; CARDOSO, L. T. S.; BARRETO, R. M. de C. S.; SOUZA, R. C. de. Perfil sociodemográfico e necessidades de educação em saúde entre cuidadores de idosos em uma unidade de saúde da família em Ilhéus, Bahia, Brasil. Revista Brasileira de Medicina de Família e Comunidade, v.8, n. 28, p.172-9, jul/set, 2013.

FARIAS, R.G.; SANTOS, S.M. A. Influência dos determinantes do envelhecimento ativo entre idosos mais idosos. Texto Contexto Enfermagem, v.21, n.1, p. 167-76, 2012. Disponível em: 
http://www.scielo.br/pdf/tce/v21n1/a19v21n1.pdf. Acesso em: 12 set 2016.

GAIOLI, C. C. L. O.; FUREGATO, A. R. F.; SANTOS, J. L. F. Perfil de cuidadores de idosos com doença de Alzheimer associado à resiliência. Texto Contexto Enfermagem, v.21, n.1, p.150-7,jan-mar, 2012. Disponível em: http://www.scielo.br/scielo.

php?script=sci_arttext\&pid=S0104-07072012000100017. Acesso em: 12 set 2016.

LANDEIRO, M. J. L.; PERES, H. H. C.; MARTINS, T.Avaliação de necessidades informacionais dos cuidadores domiciliares. Revista de Enfermagem da UFSM, v.5, n.3, p. 486- 498, 2015. Disponível em: http://periodicos.ufsm.br/reufsm/article/view/16886.

Acesso em: 14 set 2016.

SILVA, K. M.; SANTOS, S. M. A. de.; SOUZA, A. I. J. de. Reflexões sobre a necessidade do cuidado humanizado ao idoso e família. Saúde \& Transformação Social, v. 5, n.3, p. 20-24, 2014.

PEREIRA, K. C.; GUIMARÃES, F. DOS S.; ALCAUZA, M. T. R.; CAMPOS, D. A. DE.; PIRES, R. O. M. Percepção, conhecimento e habilidades de cuidadores em saúde bucal de idosos acamados.Saúde \& Transformação Social. v.5, n.2, p. 34- 41, 2014.

WHO. Goodhealthaddslifetoyears. Global brief for World Health Day 2012. 2012. Disponível em:

http://www.who.int/ageing/publications/upcoming_publications/en/index.html. Acesso em: 16 set 2016. 